\title{
Accurate Measurements of Long-Range Heteronuclear Coupling Constants from Homonuclear 2D NMR Spectra of Isotope-Enriched Proteins
}

\author{
Gaetano T. Montelione, ${ }^{*}$ Marjorie E. Winkler,$\dagger$ Peter Rauenbuehler,$\dagger$ \\ AND GERHARD WAGNER* \\ * Biophysics Research Division, The University of Michigan, Ann Arbor, Michigan 48109 \\ and $\dagger$ Department of Medicinal and Biomolecular Chemistry, Genentech, Inc., 460 Point San Bruno Blvd., \\ South San Francisco, California 94080
}

Received November 9, 1988

Long-range heteronuclear coupling constants are of high interest for studies of protein structure and dynamics. Today, typical sets of conformational constraints for protein structures consist of a large number of NOE constraints and a small number of dihedral angle constraints on $\phi$ and $\chi^{1}$ obtained from homonuclear proton-proton coupling constants. The resultant structures show well-defined overall polypeptide folds. However, local backbone structures are not determined as precisely as may be possible with additional data. Furthermore, many side chains are less well defined than the backbone. While amino acid side chains may often adopt multiple conformations, there are no good approaches for characterizing their distributions in proteins.

Measurements of heteronuclear coupling constants would provide a whole new class of constraints that could significantly improve protein structures at the local level, where improvement is needed most. The most promising approach is to measure ${ }^{15} \mathrm{~N}-{ }^{1} \mathrm{H}$ vicinal coupling constants. This would lead to the following achievements. (i) Measurements of the coupling constants between the peptide nitrogen and the $\beta$-protons, combined with measurements of homonuclear coupling constants between $\alpha$ - and $\beta$-protons, can provide a characterization of the side-chain dihedral angle $\chi^{1}$ and stereospecific assignments of $\beta$-methylene protons. For mobile side chains, these data can also be used to determine distributions of rotamer populations. This approach is an improvement over previous strategies which have used homonuclear coupling constants and NOEs to achieve stereospecific assignments (1). (ii) Stereospecific assignments of $\beta$-methylene proton resonances, in turn, would provide a more precise interpretation of intraresidue $\mathrm{H}^{\mathrm{N}}-\mathrm{H}^{\beta}$ and sequential $\mathrm{H}^{\beta}-\mathrm{H}^{\mathrm{N}}$ NOEs, which depend on the values of the intervening dihedral angles $\phi, \chi^{1}$ and $\chi^{1}, \psi$, respectively. (iii) Measurements of the coupling constants between the $\alpha$-proton of residue $i$ and the peptide nitrogen of residue $i+1$, combined with sequential NOEs, should provide improved determinations of the backbone dihedral angle $\psi_{i}$. (iv) Vicinal couplings between methylene protons and side-chain amide or guanido nitrogens could also provide $\operatorname{Asn} \chi^{2}, \operatorname{Gln} \chi^{3}$, and $\operatorname{Arg} \chi^{4}$ dihedral angle constraints. 
These motivations for measuring ${ }^{15} \mathrm{~N}-{ }^{1} \mathrm{H}$ coupling constants have long been recognized. However, it has been difficult to measure heteronuclear coupling constants accurately from NMR spectra of proteins. When the coupling constants must be measured from the multiplet structures of cross peaks in 2D heteronuclear correlation spectra the measurements are biased by cancellation or summation effects of crosspeak components (2). In addition, such experiments suffer from poor sensitivity at natural abundance ${ }^{15} \mathrm{~N}$ and are often difficult to phase. Until now, only the sensitivity problem has been solved, using technologies for uniform ${ }^{15} \mathrm{~N}$ enrichment of proteins.

This paper describes our simple approach for measuring ${ }^{15} \mathrm{~N}-{ }^{1} \mathrm{H}$ coupling constants in uniformly ${ }^{15} \mathrm{~N}$-enriched proteins. We record homonuclear $2 \mathrm{D}{ }^{1} \mathrm{H}$ NMR spectra (e.g., NOESY, ROESY, RELAY, or TOCSY) that contain cross peaks between the protons directly bound to ${ }^{15} \mathrm{~N}$ and the protons for which the long-range coupling to that same nitrogen should be measured. Especially useful are cross peaks between amide protons and the $\beta$-protons of the same residue (to characterize $\chi^{1}$ ) or cross peaks between the $\alpha$-protons of residue $i$ and the amide protons of residue $i$ +1 (to characterize $\psi$ ). Since we do not pulse the ${ }^{15} \mathrm{~N}$ nucleus the cross peaks show E.COSY-type multiplet structures where the heteronuclear couplings are passive. They are split in one dimension by the large direct heteronuclear coupling and, in the other dimension, by the small long-range heteronuclear coupling of interest. The large direct splitting provides good resolution of the cross-peak components and allows accurate measurements of both heteronuclear coupling constants. We use the cross peak for which the larger direct splitting is along $\omega_{1}$ and the smaller long-range coupling is along $\omega_{2}$ to take advantage of the higher resolution along the latter dimension. As in homonuclear E.COSY, the sign of the long-range coupling relative to the direct heteronuclear coupling can be determined. Similar effects have been reported previously for ${ }^{113} \mathrm{Cd}$-enriched metallothionein-2 (3).

As an example of this approach, we have used NOESY to measure long-range heteronuclear coupling constants in uniformly ${ }^{15} \mathrm{~N}$-enriched type $\alpha$ human transforming growth factor ( $\mathrm{hTGF} \alpha$ ). TGF $\alpha$ is a small protein containing 50 amino acid residues and three disulfide bonds. The cloned protein is expressed in Escherichia coli $(4,5)$. Its small size and biological importance make hTGF $\alpha$ an ideal system in which to develop approaches for protein structure determination and molecular design. For this reason, we have recently determined a nearly complete set of sequence-specific proton resonance assignments and have characterized the secondary structure of hTGF $\alpha$ using conventional homonuclear 2D NMR methods (6). The refinement of the three-dimensional structure of hTGF $\alpha$ will be aided greatly by measurements of heteronuclear ${ }^{15} \mathrm{~N}-{ }^{1} \mathrm{H}$ coupling constants.

Uniformly ${ }^{15} \mathrm{~N}$-enriched hTGF $\alpha$ was prepared by growing the $E$. coli expression system in a totally synthetic media containing $\left({ }^{15} \mathrm{NH}_{4}\right)_{2} \mathrm{SO}_{4}$ as the major nitrogen source. The region of cross peaks between the amide protons (along $\omega_{1}$ ) and the aliphatic protons (along $\omega_{2}$ ) of a NOESY spectrum of $99 \%{ }^{15} \mathrm{~N}$-enriched hTGF $\alpha$ in $\mathrm{H}_{2} \mathrm{O}$ solvent is shown in Fig. 1. Each NOESY cross peak involving an $\mathrm{H}^{\mathrm{N}}$ proton is split into two components in the $\omega_{1}$ dimension due to ${ }^{1} J\left({ }^{15} \mathrm{~N}-\mathrm{H}\right)$. Many also show measurable displacements in $\omega_{2}$ arising from vicinal ${ }^{15} \mathrm{~N}-{ }^{1} \mathrm{H}$ coupling. The patterns of these cross peaks show that the vicinal ${ }^{15} \mathrm{~N}-{ }^{1} \mathrm{H}$ couplings have the same sign as the direct ${ }^{15} \mathrm{~N}-{ }^{1} \mathrm{H}$ couplings because the low-field component along $\omega_{1}$ is also the low- 


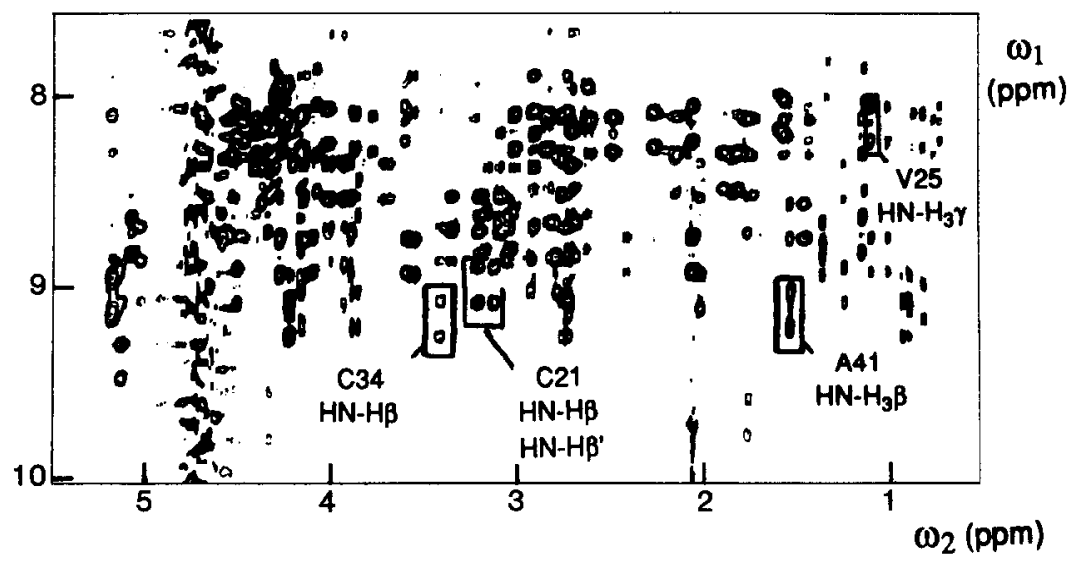

FIG. 1. Expansion of a ${ }^{1} \mathrm{H}-\mathrm{NOESY}$ spectrum of $99 \%{ }^{15} \mathrm{~N}$-enriched hTGF $\alpha$ in $90 \% \mathrm{H}_{2} \mathrm{O} / 10 \%{ }^{2} \mathrm{H}_{2} \mathrm{O}$ solvent, protein concentration about $5 \mathrm{~m} \mathrm{M}, \mathrm{pH} 3.5$, temperature $30^{\circ} \mathrm{C}$, and mixing time $350 \mathrm{~ms}$. The cross peaks between the $\mathrm{H}^{\mathrm{N}}$ and aliphatic protons are shown. The digital resolutions are $12.6 \mathrm{and} 1.0 \mathrm{~Hz} / \mathrm{pt}$ in $\omega_{1}$ and $\omega_{2}$, respectively.

field component along $\omega_{2}$. Since it is known that these direct ${ }^{15} \mathrm{~N}-{ }^{1} \mathrm{H}$ couplings are negative (7), the vicinal ${ }^{15} \mathrm{~N}-{ }^{1} \mathrm{H}$ couplings must also be negative. Expansions of representative examples are shown in Figs. 2 and 3.

Long-range heteronuclear coupling constants were determined by measuring the displacements of resonance peaks in $\omega_{2}$ cross sections. The precision of our measurements is determined both by the digital resolution of the transformed spectra (1.0 $\mathrm{Hz} / \mathrm{pt}$ in $\omega_{2}$ ) and by our ability to identify the centers of the displaced cross-peak components. Measurements made from several cross sections of the same cross peak indicate a reliability of about $\pm 0.5 \mathrm{~Hz}$. Approaches for improving this precision will be presented elsewhere.

Representative $\mathrm{H}^{\mathrm{N}}-\mathrm{H}^{\beta}$ cross peaks bearing conformational information of interest are shown in Fig. 2. For Cys-34 the ensemble-averaged ${ }^{3} J\left({ }^{15} \mathrm{~N}-\mathrm{H}^{\beta}\right)$ coupling to the downfield $\mathrm{H}^{\beta}$ proton is $-5.5 \pm 0.5 \mathrm{~Hz}$ (Fig. $2 \mathrm{~A}$ ), corresponding to a trans orientation of these two atoms $(8)$. Information is not yet available for the upfield $\mathrm{H}^{\beta}$ proton owing to partial overlap of this cross peak. For Cys-21, both $\mathrm{H}^{\mathrm{N}}-\mathrm{H}^{\beta}$ cross peaks are resolved (Fig. 2B) with ${ }^{3} J\left({ }^{15} \mathrm{~N}-\mathrm{H}^{\beta}\right)$ values of $-1.3 \pm 0.5$ and $-1.8 \pm 0.5 \mathrm{~Hz}$ for the downfield and upfield resonances, respectively. These measurements indicate $(8)$ that both protons are gauche with respect to the ${ }^{15} \mathrm{~N}$ atom; i.e., the corresponding dihedral angle $\chi^{1}$ is about $180^{\circ}$.

In order to evaluate the accuracy of these measurements, we have determined the ${ }^{3} J\left({ }^{15} \mathrm{~N}-\mathrm{H}^{\beta}\right)$ coupling constants for well-resolved intraresidue $\mathrm{H}^{\mathrm{N}}-\mathrm{H}_{3}^{\beta}$ cross peaks of Ala-41 and Ala-46. For an alanine side chain it is reasonable to assume equally populated staggered rotamer states for the methyl protons. The corresponding ensembleaveraged ${ }^{3} J\left({ }^{15} \mathrm{~N}-\mathrm{H}^{\beta}\right)$ coupling constants calculated from different published calibrations of the Karplus curve ( $8-10$ ) range from -2.1 to $-2.9 \mathrm{~Hz}$, in good agreement with our measured values of $-2.4 \pm 0.5 \mathrm{~Hz}$ for both Ala-41 (Fig. 3A) and Ala-46 (not shown). As an additional check for systematic errors, we also examined some 


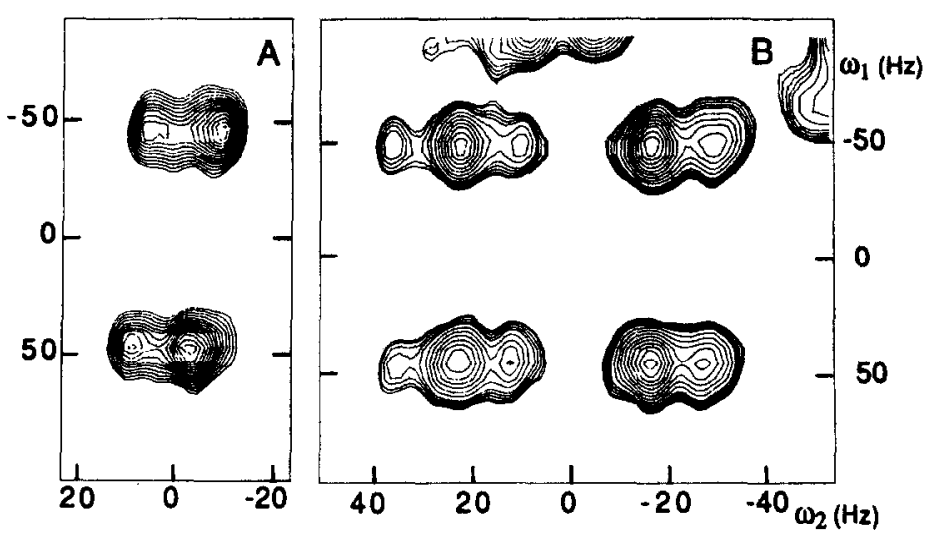

FIG. 2. Expansions of cross peaks involving methylene resonances from the NOESY spectrum of Fig. 1. (A) Intraresidue $\mathrm{H}^{\mathrm{N}}-\mathrm{H}^{\beta}$ cross peak of Cys-34 manifesting an $\omega_{2}$ displacement due to vicinal ${ }^{3} J\left({ }^{15} \mathrm{~N}-\right.$ $\mathrm{H}^{\beta}$ ) heteronuclear coupling of $-5.5 \pm 0.5 \mathrm{~Hz}$. (B) Both intraresidue ${ }^{3} J\left({ }^{15} \mathrm{~N}-\mathrm{H}^{\beta}\right)$ cross peaks of Cys-21, manifesting heteronuclear couplings of $-1.3 \pm 0.5$ and $-1.8 \pm 0.5 \mathrm{~Hz}$, respectively. These measurements indicate an ensemble-averaged dihedral angle $\chi^{\prime}$ of about $180^{\circ}$.

cross peaks in which the $\mathrm{H}^{\mathrm{N}}$ proton is coupled to ${ }^{15} \mathrm{~N}$ but the aliphatic proton is not, e.g., the intraresidue $\mathrm{H}^{\mathrm{N}}-\mathrm{H}_{3}^{\gamma}$ NOESY cross peak of Val-25 (Fig. 3B). These cross peaks show no displacement in the $\omega_{2}$ dimension.

In principle the approach described in this paper can also be used to measure vicinal ${ }^{3} J\left(\mathrm{H}^{\alpha}-{ }^{15} \mathrm{~N}\right)$ couplings as displacements in interresidue $\mathrm{H}^{\alpha}-\mathrm{H}^{\mathrm{N}}$ NOESY cross peaks. These coupling constants depend on the backbone dihedral angle $\psi$ and are proposed to range from about 0 to $-5 \mathrm{~Hz}(7,11,12)$. While only a few $\mathrm{H}^{\alpha}-\mathrm{H}^{\mathrm{N}}$ NOESY cross peaks for hTGF $\alpha$ are sufficiently well resolved to be useful in these measurements, none of these have ${ }^{3} J\left(\mathrm{H}^{\alpha}-{ }^{15} \mathrm{~N}\right)$ splittings large enough to be mea-

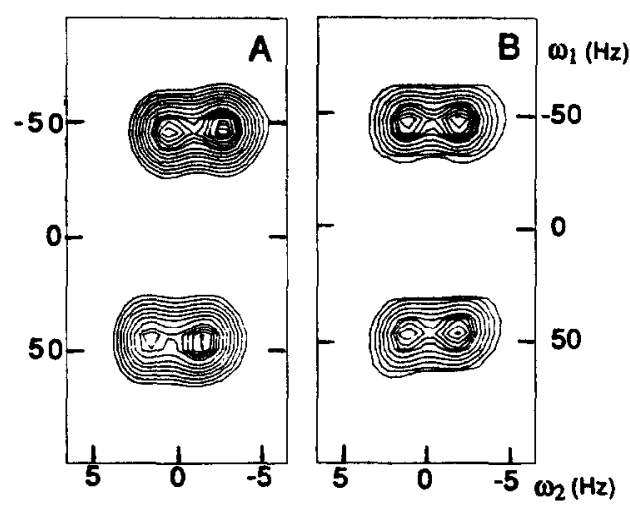

FIG. 3. Expansions of cross peaks involving methyl resonances from the NOESY spectrum of Fig. 1. (A) Intraresidue $\mathrm{H}^{\mathrm{N}}-\mathrm{H}_{3}^{\beta}$ cross peak of Ala-41. The displacement along $\omega_{2}$ is due to the vicinal ${ }^{3} J\left({ }^{15} \mathrm{~N}-\mathrm{H}^{\beta}\right)$ heteronuclear coupling constant of $-2.4 \pm 0.5 \mathrm{~Hz}$. (B) Intraresidue $\mathrm{H}^{\mathrm{N}}-\mathrm{H}_{3}^{\gamma}$ cross peak of Val-25 showing no long-range heteronuclear coupling. 
sured. They are all smaller than about $1 \mathrm{~Hz}$. Similar observations have been reported for ${ }^{15} \mathrm{~N}$-enriched peptides $(8,13)$.

To illustrate the physical basis of this method, we have used product operator formalism (14) to simulate an intraresidue $\mathbf{H}^{\mathbf{N}}-\mathrm{H}^{\beta}$ NOESY cross peak of the ${ }^{15} \mathrm{~N}$-enriched amino acid spin system shown in Fig. 4. We considered the NOESY peak at $\omega_{1}=\Omega_{\mathrm{HN}}$ and $\omega_{2}=\Omega_{\mathrm{H} \beta}$ for which the magnetization begins on the $\mathrm{H}^{\mathrm{N}}$ proton. The relevant terms of the product operator at the end of the evolution period are

$$
-I_{y} \cos \left(\Omega_{I} t_{1}\right) \cos \left(\pi J_{1} t_{1}\right) \cos \left(\pi J_{2} t_{1}\right)+2 I_{y} K_{z} \sin \left(\Omega_{I} t_{1}\right) \sin \left(\pi J_{1} t_{1}\right) \cos \left(\pi J_{2} t_{1}\right)
$$

in which $J_{1} \equiv{ }^{1} J\left({ }^{15} \mathrm{~N}-\mathrm{H}\right), J_{2} \equiv{ }^{3} J\left(\mathrm{HN}-\mathrm{H}^{\alpha}\right)$, and $I$ and $K$ refer to the $\mathrm{H}^{\mathrm{N}}$ proton and ${ }^{15} \mathrm{~N}$ nuclei, respectively. The additional terms which are not shown are eliminated by standard NOESY phase cycles. The second proton pulse of the NOESY sequence converts the in-phase and antiphase terms of Eq. [1] into longitudinal magnetization
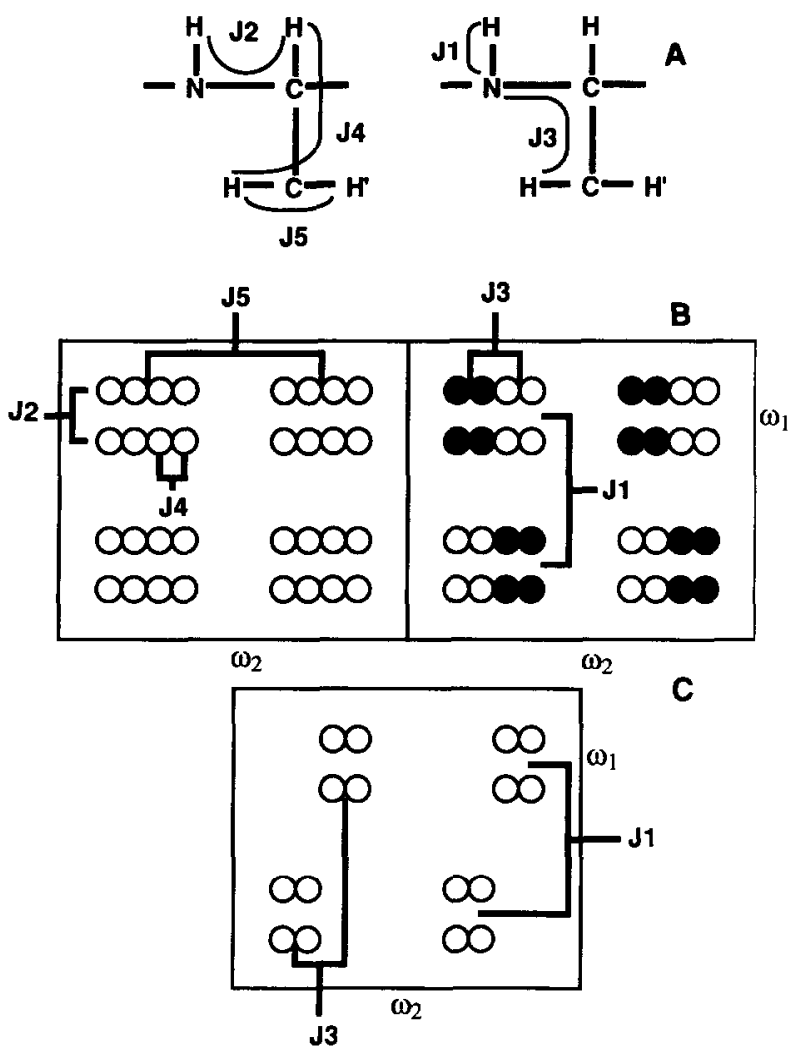

FIG. 4. Schematic drawing of a simulated $\mathrm{H}^{\mathrm{N}}-\mathrm{H}^{\beta}$ NOESY cross peak for an ${ }^{15} \mathrm{~N}$-enriched protein. (A) A simple amino acid spin system showing the homonuclear (left) and heteronuclear (right) spin coupling constants used for the cross-peak simulation. The relevant coupling constants $J_{1}, J_{2}, J_{3}, J_{4}$, and $J_{5}$ are defined in the text. In this simulation, $J_{1}>J_{5}>J_{2}=J_{3}>J_{4}$ and the product $J_{1} \times J_{3}>0$. (B) Contributions to cross-peak fine structure arising from longitudinal magnetization transfer $I_{z}$ to $M_{z}$ (left) and from transfer of heteronuclear two-spin order $I_{z} K_{z}$ to $M_{z} K_{z}$ (right) during the NOESY mixing time $\tau_{\mathrm{m}}$. (C) Superposition of these two components. 
$\left(I_{z}\right)$ and two-spin order $\left(I_{z} K_{z}\right)$, respectively. Because the ${ }^{15} \mathrm{~N}$ nuclei are not affected by this second proton pulse, the $K_{z}$ components of the product operator remain aligned along the $z$ axis. Nuclear Overhauser effects between the $\mathrm{H}^{\mathrm{N}}$ and the $\mathrm{H}^{\beta}$ protons during the mixing period next convert longitudinal magnetization and two-spin order of the $\mathrm{H}^{\mathrm{N}}$ proton into the corresponding states of the $\mathrm{H}^{\beta}$ proton. Ignoring the diagonal peaks and considering only the magnetization on the $y$ axis, the observable terms of the final two-dimensional product operator are

$$
\begin{gathered}
-M_{y} \cos \left(\Omega_{I} t_{1}\right) \cos \left(\Omega_{M} t_{2}\right) \cos \left(\pi J_{1} t_{1}\right) \cos \left(\pi J_{2} t_{1}\right) \cos \left(\pi J_{3} t_{2}\right) \cos \left(\pi J_{4} t_{2}\right) \cos \left(\pi J_{5} t_{2}\right) \\
-M_{y} \sin \left(\Omega_{I} t_{1}\right) \sin \left(\Omega_{M} t_{2}\right) \sin \left(\pi J_{1} t_{1}\right) \cos \left(\pi J_{2} t_{1}\right) \sin \left(\pi J_{3} t_{2}\right) \cos \left(\pi J_{4} t_{2}\right) \cos \left(\pi J_{5} t_{2}\right)
\end{gathered}
$$

in which $J_{3} \equiv{ }^{3} J\left({ }^{15} \mathrm{~N}-\mathrm{H}^{\beta}\right), J_{4}={ }^{2} J\left(\mathrm{H}^{\beta}-\mathrm{H}^{\beta}\right), J_{5} \equiv{ }^{3} J\left(\mathrm{H}^{\alpha}-\mathrm{H}^{\beta}\right)$, and $M$ denotes the $\mathrm{H}^{\beta}$ spin. The first term of Eq. [2] is the usual in-phase homonuclear NOESY cross peak, while the second term, arising from heteronuclear two-spin order during the mixing time, is a superimposed NOESY cross peak which is in-phase with respect to all of the homonuclear couplings and antiphase in $t_{1}$ and $t_{2}$ relative to ${ }^{1} J\left({ }^{15} \mathrm{~N}-\mathrm{H}\right)$ and ${ }^{3} J\left({ }^{15} \mathrm{~N}-\mathrm{H}^{\beta}\right)$, respectively. This second pathway of magnetization is converted back into observable magnetization only if the two protons associated with the NOESY cross peak are coupled to the same ${ }^{15} \mathrm{~N}$ nucleus (or in the special case of simultaneous ${ }^{1} \mathrm{H}-{ }^{1} \mathrm{H}$ and ${ }^{15} \mathrm{~N}-{ }^{15} \mathrm{~N}$ incoherent magnetization transfer). The cross-peak fine structures resulting from each of the two terms of Eq. [2], and their superposition, are shown in Fig. 4.

The primary limitation of the approach described here is overlap between NOESY cross peaks. Many of the NOESY cross peaks of ${ }^{15} \mathrm{~N}$-enriched hTGF $\alpha$ have one or both components overlapped with other peaks, complicating their interpretation in terms of coupling constants. In this respect, the TOCSY and RELAY-COSY versions of the experiment, which contain fewer cross peaks, are advantageous. Improved resolution could perhaps be obtained best by introducing a third heteronuclear frequency domain. This could be achieved by combining ${ }^{15} \mathrm{~N}-{ }^{1} \mathrm{H}$ heteronuclear-correlated and homonuclear TOCSY, RELAY-COSY, ROESY, or NOESY pulse sequences into heteronuclear 3D NMR experiments $(15,16)$.

The results presented in this Communication demonstrate a simple approach for measuring long-range heteronuclear couplings in ${ }^{15} \mathrm{~N}$-enriched proteins. This information can be combined with proton-proton coupling constants to determine $\beta$ methylene prochirality assignments, ensemble-averaged values of side-chain (e.g., $\left.\chi^{1}\right)$ dihedral angles, and dynamic distributions of side-chain rotomer states (13, 1719). Such information will be especially useful for refining protein structures and for characterizing subtle conformational changes which may accompany single-site mutations.

\section{ACKNOWLEDGMENTS}

We thank S. Hyberts, N. R. Nirmala, and V. Thanabal for many helpful discussions. This work was supported by Grant 1GM38608-01A1 from the National Institute of General Medical Sciences, Grant DMB86-16059 from the National Science Foundation, and by the Damon Runyon-Walter Winchell Cancer Research Fund, DRG-920. 


\section{REFERENCES}

1. S. G. HyberTs, W. MÄRKI, AND G. WAGNER, Eur. J. Biochem. 164, 625 (1987).

2. D. Neuhaus, G. WAGNER, M. VASAK, J. H. R. KäGI, AND K. WÜTHRICH, Eur. J. Biochem. 151, $257(1985)$

3. D. Neuhaus, G. Wagner, M. Vasak, J. H. R. KäGi, and K. Wüthrich, Eur. J. Biochem. 143, 659 (1984).

4. R. Derynck, A. B. Roberis, M. E. Winkier, E. Y. Chen, ANd D. V. Goeddel, Cell 38, 287 (1984).

5. M. E. WinkLer, T. Bringman, AND B. J. Marks, J. Biol. Chem. 261, 13,838 (1986).

6. G. T. Montelione, M. E. WiNKL.EK, L. E. Burion, E. RinderkNeCHI, M. B. SPORN, AND G. WAGNER, Proc. Natl. Acad. Sci. USA, in press.

7. V. F. BYSTROV, Prog. NMR Spectrosc. 10, 41 (1976).

8. A. DEMARCO, M. LliNAS, AND K. WÜTHRICH, Biopolymers 17, 2727 (1978).

9. R. L. LICHTER AND J. D. ROBERTS, J. Org. Chem. 35, 2806 (1970).

10. J. A. SOGN, W. A. GIBBONS, AND E. W. RANDAll, Biochemistry 12, 2100 (1973).

11. M. Barfield AND H. L. Gearhart, Mol. Phys. 27, 899 (1974).

12. V. F. Bystrov, Yu. D. Gavrilov, AND V. M. SolKan, J. Magn. Reson. 19, 123 (1975).

13. E. R. Stimson, Y. C. Meinwald, G. T. Montelione, and H. A. Scheraga, Int. J. Pept. Protein Res. 27, 569 (1986).

14. O. W. Sørensen, G. W. Eich, M. H. Levitt, G. Bodenhausen, AND R. R. ERnSt, Prog. NMR Spectrosc. 16, 163 (1983).

15. C. GRiesinger, O. W. SøRensen, AND R. R. ERnSt, J. Am. Chem. Soc. 109, 7227 (1987).

16. S. W. FESIK AND E. R. P. ZUIDERWEG, J. Magn. Reson. 78, 588 (1988).

17. A. J. Fischman, H. R. Wyssbrod, W. C. Agosta, AND D. Cowburn, J. Am. Chem. Soc. 100, 54 (1978).

18. A. J. Fischman, D. H. Live, H. R. Wyssbrod, W. C. Agosta, and D. Cowburn, J. Am. Chem. Soc. 102, $2533(1980)$.

19. H. KeSSLER, C. Griesinger, AND K. WAGNER, J. Am. Chem. Soc. 109, 6927 (1987). 